PROCEEDINGS OF THE

AMERICAN MATHEMATICAL SOCIETY

Volume 131, Number 8 , Pages $2467-2478$

S 0002-9939(02)06769-2

Article electronically published on November 13, 2002

\title{
APPROXIMATION METHODS FOR NONLINEAR OPERATOR EQUATIONS
}

\author{
C. E. CHIDUME AND H. ZEGEYE
}

(Communicated by Joseph A. Ball)

\begin{abstract}
Let $E$ be a real normed linear space and $A: E \rightarrow E$ be a uniformly quasi-accretive map. For arbitrary $x_{1} \in E$ define the sequence $x_{n} \in E$ by $x_{n+1}:=x_{n}-\alpha_{n} A x_{n}, n \geq 1$, where $\left\{\alpha_{n}\right\}$ is a positve real sequence satisfying the following conditions: (i) $\sum \alpha_{n}=\infty$; (ii) $\lim \alpha_{n}=0$. For $x^{*} \in N(A):=$ $\{x \in E: A x=0\}$, assume that $\sigma:=\inf _{n \in N_{0}} \frac{\psi\left(\left\|x_{n+1}-x^{*}\right\|\right)}{\left\|x_{n+1}-x^{*}\right\|}>0$ and that $\left\|A x_{n+1}-A x_{n}\right\| \rightarrow 0$, where $N_{0}:=\{n \in N$ (the set of all positive integers): $\left.x_{n+1} \neq x^{*}\right\}$ and $\psi:[0, \infty) \rightarrow[0, \infty)$ is a strictly increasing function with $\psi(0)=0$. It is proved that a Mann-type iteration process converges strongly to $x^{*}$. Furthermore if, in addition, $A$ is a uniformly continuous map, it is proved, without the condition on $\sigma$, that the Mann-type iteration process converges strongly to $x^{*}$. As a consequence, corresponding convergence theorems for fixed points of hemi-contractive maps are proved.
\end{abstract}

\section{INTRODUCTION}

Let $E$ be a real normed linear space with dual $E^{*}$. We denote by $J$ the normalized duality mapping from $E$ to $2^{E^{*}}$ defined by

$$
J x=\left\{f^{*} \in E^{*}:\left\langle x, f^{*}\right\rangle=\|x\|^{2}=\left\|f^{*}\right\|^{2}\right\},
$$

where $\langle.,$.$\rangle denotes the generalized duality pairing. It is well known that if E^{*}$ is strictly convex, then $J$ is single-valued and if $E^{*}$ is uniformly convex, then $J$ is uniformly continuous on bounded subsets of $E$.

A map $A: D(A) \subseteq E \rightarrow E$ is called strongly accretive if for each $x, y \in D(A)$, there exist $j(x-y) \in J(x-y)$ and a real number $0<k<1$ such that

$$
\langle A x-A y, j(x-y)\rangle \geq k\|x-y\|^{2},
$$

and it is called $\phi$-strongly accretive if $\forall x, y \in D(A)$, there exist $j(x-y) \in J(x-y)$ and a strictly increasing function $\phi:[0, \infty) \rightarrow[0, \infty)$ with $\phi(0)=0$ such that

$$
\langle A x-A y, j(x-y)\rangle \geq \phi(\|x-y\|)\|x-y\| .
$$

Received by the editors December 8, 2001 and, in revised form, March 18, 2002.

2000 Mathematics Subject Classification. Primary 47H04, 47H06, 47H30, 47J05, 47J25.

Key words and phrases. Bounded operators, nonexpansive retraction, uniformly accretive maps, uniformly pseudocontractive maps, uniformly smooth Banach spaces.

The second author undertook this work with the support of the "ICTP Programme for Training and Research in Italian Laboratories, Trieste, Italy". 
The map $A$ is called uniformly accretive if $\forall x, y \in D(A)$, there exist $j(x-y) \in$ $J(x-y)$ and a strictly increasing function $\psi:[0, \infty) \rightarrow[0, \infty)$ with $\psi(0)=0$ such that

$$
\langle A x-A y, j(x-y)\rangle \geq \psi(\|x-y\|) .
$$

Closely related to the class of accretive operators is the class of pseudocontractive maps. A map $T: D(T) \subseteq E \rightarrow E$ is called strongly pseudocontractive if for each $x, y \in D(T)$, there exist $j(x-y) \in J(x-y)$ and a real number $0<s<1$ such that

$$
\langle T x-T y, j(x-y)\rangle \leq s\|x-y\|^{2},
$$

and it is called $\beta$-strongly pseudocontractive if $\forall x, y \in D(T)$, there exist $j(x-y) \in$ $J(x-y)$ and a strictly increasing function $\beta:[0, \infty) \rightarrow[0, \infty)$ with $\beta(0)=0$ such that

$$
\langle T x-T y, j(x-y)\rangle \leq\|x-y\|^{2}-\beta(\|x-y\|)\|x-y\| .
$$

The map $T$ is called uniformly pseudocontractive if $\forall x, y \in D(T)$, there exist $j(x-y) \in J(x-y)$ and a strictly increasing function $\Omega:[0, \infty) \rightarrow[0, \infty)$ with $\Omega(0)=0$ such that

$$
\langle T x-T y, j(x-y)\rangle \leq\|x-y\|^{2}-\Omega(\|x-y\|) .
$$

Observe that $T$ is uniformly pseudocontractive if and only if $(I-T)$ is uniformly accretive (where $I$ denotes the identity operator). Furthermore, a close look at the above definitions shows that the class of uniformly pseudocontractive maps includes several important classes of operators studied by various authors.

(1) If, for example, in (1.4) $\Omega(t):=s t^{2}, 0<s<1$, then we have the class of strongly pseudocontractive maps studied by several authors (see, for example [3, 4, 5, 6, 7, 8, 9, 10, 15, 16, 17, 18, 21]).

(2) If $\Omega(t)=t \beta(t)$, where $\beta:[0, \infty) \rightarrow[0, \infty)$ is a strictly increasing function with $\beta(0)=0$, then (1.4) reduces to (1.3) and we have the class of $\beta$-strongly pseudocontractive maps studied by several authors (see, for example [11, 12, 14. 25, 29, 30]).

Thus, we observe that the class of uniformly pseudocontractive maps includes the classes of $\beta$-strongly pseudocontractive and hence strongly pseudocontractive maps. This inclusion is proper (see [24).

Let $N(A):=\{x \in D(A): A x=0\}$ and $F(T):=\{x \in D(T): T x=x\}$ denote the null space of $A$ and the fixed-point set of $T$, respectively. If $N(A) \neq \emptyset$ (respectively, $F(T) \neq \emptyset$ ) and (1.2) (respectively, (1.4)) holds $\forall x \in D(A)$ (respectively, $\forall x \in$ $D(T)$ ) and $y \in N(A)$ (respectively, $y \in F(T)$ ), then $A$ (respectively, $T$ ) is called uniformly quasi-accretive (respectively, uniformly hemi-contractive). We observe that a uniformly quasi-accretive map has at most one solution $x^{*}$. This follows from (1.2).

The accretive operators were introduced independently in 1967 by Browder [2] and Kato [20. Interest in such maps stems mainly from their firm connection with equations of evolution. It is known (see, e.g., 31]) that many physically significant problems can be modeled by initial-value problems of the form

$$
x^{\prime}(t)+A x(t)=0, x(0)=x_{0},
$$

where $A$ is an accretive operator in an appropriate Banach space. Typical examples where such evolution equations occur can be found in the heat, wave or Schrödinger equations. The solutions of the equation $A x=0$ are precisely the equilibrium 
points of the system (1.5). Consequently, considerable research efforts have been devoted, especially within the past 20 years or so, to methods of approximating these equilibrium points (when they exist). Clearly a map $T$ is pseudocontractive if and only if $A:=I-T$ is accretive so that solutions of $A x=0$ for accretive operator $A$ correspond to fixed points of $T$.

In 1987, in this connection, one of the authors (CEC) (6 ) proved that, if $X=$ $L_{p}(p \geq 2), K$ is a bounded closed convex nonempty subset of $X$ and $T: K \rightarrow K$ is Lipschitzian and strongly pseudocontractive, then the Mann iteration process (see, e.g., 23]) converges to the fixed points of $T$. In 1990, he extended the result ([7) to $L_{p}\left(\right.$ or $\left.l_{p}\right)$ spaces, $1<p<\infty$, using different conditions on the $\left\{\alpha_{n}\right\}$ and a different proof technique (see, e.g., a recent survey by B. E. Rhoades and L. Saliga [28] and the reference therein). These results which generalize several important known results have themselves been extended to various Banach spaces and to various classes of nonlinear operators (see, e.g., [11, 12, 14, 16, 17, 18, 24, 29]). The class of uniformly hemi-contractive operators and, correspondingly, the class of uniformly quasi-accretive operators now seem to be the largest class for which solutions are unique and convergence theorems have been proved.

In this connection, Osilike 26, 27] observed that the class of maps studied by Dunn [18] and Weng [30] are proper subclasses of the class of uniformly hemicontractive maps and proved that if $K$ is a nonempty subset of a uniformly smooth Banach space and $T: K \rightarrow 2^{K}$ is a multi-valued uniformly hemi-contractive map, then a Mann-type iteration sequence converges strongly to the fixed point of $T$. In 29], Zhang proved that if $D$ is a nonempty subset of a real smooth Banach space $E$, and $T: D \rightarrow 2^{E}$ is a uniformly hemi-contractive map such that $T(D):=\bigcup_{x \in D} T x$ is a bounded subset of $E$, then an Ishikawa-type sequence (see, e.g., [19, 29]) converges strongly to $x^{*} \in F(T)$, provided that the sequence is well defined $\forall n \geq 0, \| \eta_{n}-$ $\xi_{n+1} \| \rightarrow 0$ and $\sigma:=\inf _{n \in N_{0}} \frac{\psi\left(\left\|x_{n+1}-x^{*}\right\|\right)}{\left\|x_{n+1}-x^{*}\right\|^{2}}>0$, where $\eta_{n} \in T y_{n}, \xi_{n+1} \in T x_{n+1}$, $N_{0}:=\left\{n \in N\right.$ (the set of all positive integers) $\left.: x_{n+1} \neq x^{*}\right\}$ and $\psi:[0, \infty) \rightarrow$ $[0, \infty)$ is a strictly increasing function with $\psi(0)=0$. Recently, Moore and Nnoli 24] proved that if $E$ is a real normed linear space and $T: E \rightarrow 2^{E}$ is a uniformly continuous and uniformly hemi-contractive set-valued map with nonempty closed values such that the range of $T$ is bounded, then an Ishikawa-type iteration process converges strongly to $x^{*} \in F(T)$. Of course, the uniform continuity assumption is stronger than the condition $\left\|\eta_{n}-\xi_{n+1}\right\| \rightarrow 0, \eta_{n} \in T y_{n}, \xi_{n+1} \in T x_{n+1}$ imposed in [29].

It is our purpose in this paper to prove that a Mann-type iteration process converges strongly to the zero of a uniformly quasi-accretive map in arbitrary normed linear spaces. Related results deal with the strong convergence of this iteration process to fixed points of uniformly hemi-contractive maps in arbitrary real normed linear spaces. Our theorems unify and extend the results of several authors. In particular, our Corollary 3.3 (below) extends the results of Zhang from real smooth Banach spaces to arbitrary normed linear spaces and without the requirement that $T(D)$ be bounded. In addition, our Theorem 3.6 (below) dispenses with the requirement imposed by Moore and Nnoli that the range of $T$ be bounded. Furthermore, our theorems are proved for the Mann-type iteration process which has a much simpler recursion formula and hence is more convenient than the Ishikawa formula in applications. Finally, we remark that all our theorems hold for the so-called Mann-type sequences with errors (see, e.g., [21] and our Remarks 3.12-3.14 below). 


\section{Preliminaries}

Let $E$ be a real normed linear space with dual $E^{*}$. The modulus of smoothness of $E$ is defined by

$$
\rho_{E}(\tau):=\sup \left\{\frac{\|x+y\|+\|x-y\|}{2}-1:\|x\|=1,\|y\|=\tau\right\}, \tau>0 .
$$

$E$ is said to be uniformly smooth if $\lim \frac{\rho_{E}(\tau)}{\tau}=0$. Typical examples of such spaces are the Lebesgue $L_{p}$, the sequence $l_{p}$, and the Sobolev $W_{p}^{m}$ spaces, $1<p<\infty$.

A map $P$ of $E$ to $E$ is said to be a retraction if $P^{2}=P$. A subset $K$ of $E$ is said to be a nonexpansive retract of $E$ if there exists a nonexpansive retraction of $E$ onto $K$. If $E=H$ is a Hilbert space, the metric projection $P_{K}$ is a nonexpansive retraction from $H$ to any closed convex subset $K$ of $H$.

In the sequel we shall use the following two well-known lemmas.

Lemma 2.1 (see, e.g., [14]). Let $E$ be a real normed linear space and $J$ the normalized duality map on $E$. Then for any given $x, y \in E$, the following inequality holds:

$$
\|x+y\|^{2} \leq\|x\|^{2}+2\langle y, j(x+y)\rangle, \forall j(x+y) \in J(x+y) .
$$

Lemma $2.2([1])$. Let $\left\{\lambda_{n}\right\}$ and $\left\{\gamma_{n}\right\}$ be sequences of nonnegative numbers and $\left\{\alpha_{n}\right\}$ be a sequence of positive numbers satisfying the conditions $\sum_{1}^{\infty} \alpha_{n}=\infty$ and $\frac{\gamma_{n}}{\alpha_{n}} \rightarrow 0$, as $n \rightarrow \infty$. Let the recursive inequality

$$
\lambda_{n+1} \leq \lambda_{n}-\alpha_{n} \psi\left(\lambda_{n}\right)+\gamma_{n}, n=1,2, \ldots,
$$

be given where $\psi:[0, \infty) \rightarrow[0, \infty)$ is a strictly increasing function such that it is positive on $(0, \infty)$ and $\psi(0)=0$. Then $\lambda_{n} \rightarrow 0$, as $n \rightarrow \infty$.

\section{MAin RESUlts}

We first prove the following technical lemma which will be used in the sequel.

Lemma 3.1. Let $\left\{\lambda_{n}\right\}$ and $\left\{b_{n}\right\}$ be sequences of nonnegative numbers and $\left\{\alpha_{n}\right\} \subseteq$ $(0,1)$ a sequence satisfying the conditions that $\left\{\lambda_{n}\right\}$ is bounded, $\sum_{1}^{\infty} \alpha_{n}=\infty$ and $b_{n} \rightarrow 0$, as $n \rightarrow \infty$. Let the recursive inequality

$$
\lambda_{n+1}^{2} \leq \lambda_{n}^{2}-2 \alpha_{n} \psi\left(\lambda_{n+1}\right)+2 \alpha_{n} b_{n} \lambda_{n+1}, n=1,2, \ldots
$$

be given where $\psi:[0, \infty) \rightarrow[0, \infty)$ is a strictly increasing function such that it is positive on $(0, \infty)$ and $\psi(0)=0$. Then $\lambda_{n} \rightarrow 0$, as $n \rightarrow \infty$.

Proof. Let $m>0$ be such that $\lambda_{n} \leq m, \forall n \geq 1$. Assume that $\lim \inf \lambda_{n}=a \geq 0$.

Claim: $a=0$. Suppose not. Then there exists an integer $N_{1}>0$ such that $\lambda_{n} \geq \frac{a}{2} \forall n \geq N_{1}$. Since $b_{n} \rightarrow 0$, there exists $N_{2}>0$ such that $b_{n} \leq \frac{\psi\left(\frac{a}{2}\right)}{2 m}$ for $n \geq N_{2}$. Now let $n \geq N:=\max \left\{N_{1}, N_{2}\right\}$. Then we have from (3.1) that

$$
\lambda_{n+1}^{2} \leq \lambda_{n}^{2}-2 \alpha_{n} \psi\left(\frac{a}{2}\right)+\alpha_{n} \psi\left(\frac{a}{2}\right) \leq \lambda_{n}^{2}-\alpha_{n} \psi\left(\frac{a}{2}\right), \forall n>N
$$

which implies that $\sum \alpha_{n}<\infty$, a contradiction. Therefore, $a=0$. Thus, there exists a subsequence $\left\{\lambda_{n_{j}}\right\}$ of $\left\{\lambda_{n}\right\}$ such that $\lim \lambda_{n_{j}}=0$. For arbitrary $\epsilon>0$, let $N_{3}>0$ be such that $\lambda_{n_{j}}<\frac{\epsilon}{4} \forall j \geq N_{3}$ and $N_{4}>0$ be such that $b_{n} \leq \frac{\psi\left(\frac{\epsilon}{4}\right)}{2 m}$. Let $N_{*}:=\max \left\{N_{3}, N_{4}\right\}$ and fix $j_{*}>N_{*}$. Then we show that $\lambda_{n_{j_{*}}+k}<\frac{\epsilon}{4} \forall k \in N$. For $k=0$, the result clearly holds. Suppose it holds for any $k>0$. Then we show that 
it holds for $k+1$. Suppose not. Then we have $\lambda_{n_{j_{*}}+k+1} \geq \frac{\epsilon}{4}$. Thus, from (3.1) and the above estimates we get that

$$
\begin{aligned}
\frac{\epsilon^{2}}{16} \leq \lambda_{n_{j_{*}}+k+1}^{2} & \leq \lambda_{n_{j_{*}+k}}^{2}-2 \alpha_{n_{j_{*}}+k} \psi\left(\lambda_{n_{j_{*}}+k+1}\right)+2 \alpha_{n_{j_{*}}+k} b_{n_{j_{*}}+k} \lambda_{n_{j_{*}}+k+1} \\
& \leq \lambda_{n_{j_{*}+k}}^{2}-2 \alpha_{n_{j_{*}}+k} \psi\left(\frac{\epsilon}{4}\right)+\alpha_{n_{j_{*}}+k} \psi\left(\frac{\epsilon}{4}\right) \leq \lambda_{n_{j_{*}}+k}^{2}<\frac{\epsilon^{2}}{16}
\end{aligned}
$$

a contradiction. Therefore, $\lambda_{n_{j_{*}}+k}<\frac{\epsilon}{4} \forall k \in N$ and hence $\lambda_{n} \rightarrow 0$ as $n \rightarrow \infty$.

Theorem 3.2. Let $E$ be a real normed linear space. Suppose $A: E \rightarrow E$ is a uniformly quasi-accretive map. For arbitrary $x_{1} \in E$, define the sequence $\left\{x_{n}\right\}$ iteratively by

$$
x_{n+1}:=x_{n}-\alpha_{n} A x_{n}, n \geq 1,
$$

where $\lim _{n \rightarrow \infty} \alpha_{n}=0$ and $\sum \alpha_{n}=\infty$. For $x^{*} \in N(A)$, suppose that $\sigma:=$ $\inf _{n \in N_{0}} \frac{\psi\left(\left\|x_{n+1}-x^{*}\right\|\right)}{\left\|x_{n+1}-x^{*}\right\|}>0$ and that $\left\|A x_{n+1}-A x_{n}\right\| \rightarrow 0$, where $N_{0}:=\{n \in N:$ $\left.x_{n+1} \neq x^{*}\right\}$ and $\psi:[0, \infty) \rightarrow[0, \infty)$ is a strictly increasing function with $\psi(0)=0$. Then $\left\{x_{n}\right\}$ converges strongly to the unique solution of the equation $A x=0$.

Proof. By the recursion formula (3.2) and Lemma 2.1 we have that

$$
\begin{aligned}
& \left\|x_{n+1}-x^{*}\right\|^{2} \\
& \quad=\left\|x_{n}-x^{*}-\alpha_{n} A x_{n}\right\|^{2} \leq\left\|x_{n}-x^{*}\right\|^{2}-2 \alpha_{n}\left\langle A x_{n}, j\left(x_{n+1}-x^{*}\right)\right\rangle \\
& \quad=\left\|x_{n}-x^{*}\right\|^{2}-2 \alpha_{n}\left\langle A x_{n+1}, j\left(x_{n+1}-x^{*}\right)\right\rangle-2 \alpha_{n}\left\langle A x_{n}-A x_{n+1}, j\left(x_{n+1}-x^{*}\right)\right\rangle \\
& \quad \leq\left\|x_{n}-x^{*}\right\|^{2}-2 \alpha_{n} \psi\left(\left\|x_{n+1}-x^{*}\right\|\right)+2 \alpha_{n}\left\|A x_{n+1}-A x_{n}\right\|\left\|x_{n+1}-x^{*}\right\| .
\end{aligned}
$$

If there exists an integer $n>0$ such that $x_{n+1}=x^{*}$, then we are done. Suppose $x_{n+1} \neq x^{*} \forall n \geq 1$. Then from (3.3) and our hypothesis we have that

$$
\begin{aligned}
& \left\|x_{n+1}-x^{*}\right\|^{2} \\
& \quad \leq\left\|x_{n}-x^{*}\right\|^{2}-2 \alpha_{n}\left(\frac{\psi\left(\left\|x_{n+1}-x^{*}\right\|\right)}{\left\|x_{n+1}-x^{*}\right\|}-\left\|A x_{n+1}-A x_{n}\right\|\right)\left\|x_{n+1}-x^{*}\right\| \\
& \quad \leq\left\|x_{n}-x^{*}\right\|^{2}-2 \alpha_{n}\left(\sigma-\left\|A x_{n+1}-A x_{n}\right\|\right)\left\|x_{n+1}-x^{*}\right\| .
\end{aligned}
$$

Let $N^{\prime} \subset N$ be such that $\left\|A x_{n+1}-A x_{n}\right\| \leq \sigma \forall n \geq N^{\prime}$. Then from (3.4), for all $n \geq N^{\prime}$, we get that

$$
\left\|x_{n+1}-x^{*}\right\|^{2} \leq\left\|x_{n}-x^{*}\right\|^{2} .
$$

Thus the sequence $\left\{\left\|x_{n+1}-x^{*}\right\|\right\}$ is bounded. Now let $\lambda_{n}:=\left\|x_{n}-x^{*}\right\|, b_{n}:=$ $\left\|A x_{n+1}-A x_{n}\right\|$. Then, (3.3) gives that

$$
\lambda_{n+1}^{2} \leq \lambda_{n}^{2}-2 \alpha_{n} \psi\left(\lambda_{n+1}\right)+2 \alpha_{n} b_{n} \lambda_{n+1} .
$$

The conclusion now follows from Lemma 3.1. 
Corollary 3.3. Suppose $K$ is a closed convex subset of a real normed linear space E. Suppose $T: K \rightarrow K$ is a uniformly hemi-contractive map. For arbitrary $x_{1} \in K$ define the sequence $\left\{x_{n}\right\}$ iteratively by

$$
x_{n+1}:=x_{n}-\alpha_{n}(I-T) x_{n}, n \geq 1,
$$

where $\left\{\alpha_{n}\right\} \subseteq(0,1), \lim \alpha_{n}=0$ and $\sum \alpha_{n}=\infty$. For $x^{*} \in F(T)$, suppose that $\sigma:=\inf _{n \in N_{0}} \frac{\psi\left(\left\|x_{n+1}-x^{*}\right\|\right)}{\left\|x_{n+1}-x^{*}\right\|}>0$ and that $\left\|A x_{n+1}-A x_{n}\right\| \rightarrow 0$, where $A:=I-T$, and $N_{0}, \psi$ are as in Theorem 3.2. Then $\left\{x_{n}\right\}$ converges strongly to the unique fixed point of $T$.

Proof. Observe that the fixed point of $T$ is the solution of the equation $A x=0$ and hence the proof follows as in the proof of Theorem 3.2.

Theorem 3.4. Let $E$ be a real normed linear space. Suppose $A: E \rightarrow E$ is a strongly accretive map such that the solution $x^{*}$ of the equation $A x=0$ exists. For arbitrary $x_{1} \in E$ define the sequence $\left\{x_{n}\right\}$ iteratively by

$$
x_{n+1}:=x_{n}-\alpha_{n} A x_{n}, n \geq 1 \text {, }
$$

where $\left\{\alpha_{n}\right\}$ is a positive real sequence such that $\lim \alpha_{n}=0$ and $\sum \alpha_{n}=\infty$. Suppose that $\left\|A x_{n}-A x_{n+1}\right\| \rightarrow 0$ as $n \rightarrow \infty$. Then $\left\{x_{n}\right\}$ converges strongly to the unique solution of the equation $A x=0$.

Proof. As in the proof of Theorem 3.2 and using the definition of a strongly accretive map we get that

$$
\begin{aligned}
\left\|x_{n+1}-x^{*}\right\|^{2} \leq & \left\|x_{n}-x^{*}\right\|^{2}-2 \alpha_{n} k\left\|x_{n+1}-x^{*}\right\|^{2} \\
& +2 \alpha_{n}\left\|A x_{n+1}-A x_{n}\right\|\left\|x_{n+1}-x^{*}\right\| .
\end{aligned}
$$

Now, we show that $\left\{\left\|x_{n}-x^{*}\right\|\right\}$ is bounded. Since $\left\|A x_{n+1}-A x_{n}\right\| \rightarrow 0$, there exists $M>0$ such that $\left\|A x_{n+1}-A x_{n}\right\| \leq M$ for all $n \geq 1$. If there exists $n_{0}$ such that $x_{n_{0}+1}=x^{*}$, then $x_{n}=x^{*}$ for all integers $n \geq n_{0}$ and so $\left\{\left\|x_{n}-x^{*}\right\|\right\}$ is bounded. Assume now that $\left\|x_{n+1}-x^{*}\right\| \neq 0$ for all integers $n \geq 1$. Consider the following sets: $N_{1}:=\left\{n \in N:\left\|x_{n+1}-x^{*}\right\| \leq\left\|x_{n}-x^{*}\right\|\right\}$ and $N_{2}:=\{n \in N$ : $\left.\left\|x_{n+1}-x^{*}\right\|>\left\|x_{n}-x^{*}\right\|\right\}$. Observe that $N=N_{1} \cup N_{2}$.

Case 1. If $n \in N_{1}$ for all positive integers $n \geq 1$, then $\left\|x_{n+1}-x^{*}\right\| \leq\left\|x_{1}-x^{*}\right\|$. Otherwise, if $n_{0}$ is the first positive integer in $N_{1}$, then $\left\|x_{n+1}-x^{*}\right\| \leq \| x_{n_{0}}-$ $x^{*} \| \forall n \in N_{1}$. Hence for all $n \in N_{1}$, we have

$$
\left\|x_{n+1}-x^{*}\right\| \leq \max \left\{\left\|x_{n_{0}}-x^{*}\right\|,\left\|x_{1}-x^{*}\right\|\right\} .
$$

Case 2. If $n \in N_{2}$, using inequality (3.5) we obtain that $\left\|x_{n+1}-x^{*}\right\| \leq \frac{M}{k}$ for all integers $n \in N_{2}$. Hence for all positive integers $n$,

$$
\left\|x_{n+1}-x^{*}\right\| \leq \max \left\{\left\|x_{n_{0}}-x^{*}\right\|,\left\|x_{1}-x^{*}\right\|, \frac{M}{k}\right\}
$$

and so $\left\{\left\|x_{n}-x^{*}\right\|\right\}$ is bounded. Therefore, the conclusion follows from Lemma 3.1 .

Using the method of proof of Theorem 3.4 the following generalization of Theorem 3.4 is easily proved. In fact, we have the following theorem. 
Theorem 3.5. Let $E$ be a real normed linear space. Suppose $A: E \rightarrow E$ is a $\phi$-strongly accretive map such that the solution $x^{*}$ of the equation $A x=0$ exists. For arbitrary $x_{1} \in E$ define the sequence $\left\{x_{n}\right\}$ iteratively by

$$
x_{n+1}:=x_{n}-\alpha_{n} A x_{n}, n \geq 1 \text {, }
$$

where $\left\{\alpha_{n}\right\}$ is a positive real sequence such that $\lim \alpha_{n}=0$ and $\sum \alpha_{n}=\infty$. Suppose that $\left\|A x_{n}-A x_{n+1}\right\| \rightarrow 0$ as $n \rightarrow \infty$. Then $\left\{x_{n}\right\}$ converges strongly to the unique solution of the equation $A x=0$.

Theorem 3.6. Let $E$ be a real normed linear space. Suppose $A: E \rightarrow E$ is a uniformly quasi-accretive and uniformly continuous map. For arbitrary $x_{1} \in E$ define the sequence $\left\{x_{n}\right\}$ iteratively by

$$
x_{n+1}:=x_{n}-\alpha_{n} A x_{n}, n \geq 1,
$$

where $\lim \alpha_{n}=0$ and $\sum \alpha_{n}=\infty$. Then, there exists a constant $d_{0}>0$ such that if $0<\alpha_{n} \leq d_{0},\left\{x_{n}\right\}$ converges strongly to the unique solution of the equation $A x=0$.

Proof. Let $x^{*} \in N(A)$. Since $A$ is uniformly continuous, it is bounded. Let $r>0$ be sufficiently large such that $x_{1} \in \overline{B_{r}\left(x^{*}\right)}=: B$. Then we have that $A(B)$ is bounded. Let $M=\sup \{\|A x\|: x \in B\}$ and $\psi:[0, \infty) \rightarrow[0, \infty)$ with $\psi(0)=0$ be a strictly increasing function which corresponds to $A$. As $A$ is uniformly continuous on $E$, for $\epsilon:=\frac{\psi(r)}{4 r}$ there exists a $\delta>0$ such that $x, y \in D(A),\|x-y\|<\delta$ implies $\|A(x)-A(y)\|<\epsilon$. Let $d_{0}:=\min \left\{\frac{r}{M}, \frac{\delta}{2 M}\right\}$.

Claim: $x_{n} \in B \forall n \geq 1$. We show this by induction. By our choice $x_{1} \in B$. Suppose $x_{n} \in B$. We show that $x_{n+1} \in B$. Suppose not; then $\left\|x_{n+1}-x^{*}\right\|>r$ and from (3.6) and the above estimates we have $\left\|x_{n+1}-x_{n}\right\| \leq \alpha_{n}\left\|A x_{n}\right\| \leq \alpha_{n} M<\delta$ and hence $\left\|A x_{n+1}-A x_{n}\right\|<\epsilon$. Moreover, $\left\|x_{n+1}-x^{*}\right\| \leq\left\|x_{n}-x^{*}\right\|+\alpha_{n}\left\|A x_{n}\right\| \leq$ $r+\alpha_{n} M \leq 2 r$.

Now, as in the proof of Theorem 3.2, by Lemma 2.1 and the above estimates we have that

$$
\begin{aligned}
& \left\|x_{n+1}-x^{*}\right\|^{2} \\
& \quad \leq\left\|x_{n}-x^{*}\right\|^{2}-2 \alpha_{n} \psi\left(\left\|x_{n+1}-x^{*}\right\|\right)+2 \alpha_{n}\left\|A x_{n+1}-A x_{n}\right\|\left\|x_{n+1}-x^{*}\right\| \\
& \quad \leq\left\|x_{n}-x^{*}\right\|^{2}-2 \alpha_{n} \psi(r)+\alpha_{n} \psi(r) \leq\left\|x_{n}-x^{*}\right\|^{2}-\alpha_{n} \psi(r)<r^{2},
\end{aligned}
$$

and hence $\left\|x_{n+1}-x^{*}\right\|<r$, a contradiction. Therefore, the claim holds. Now we show that $x_{n} \rightarrow x^{*}$. Since $\left\|x_{n+1}-x_{n}\right\| \rightarrow 0$, by the uniform continuity of $A$ we have $\left\|A x_{n+1}-A x_{n}\right\| \rightarrow 0$ as $n \rightarrow \infty$. The conclusion now follows from the first inequality of (3.7) with the use of Lemma 3.1.

Corollary 3.7. Suppose $K$ is a closed convex subset of a real normed linear space $E$. Suppose $T: K \rightarrow K$ is a uniformly hemi-contractive and uniformly continuous map. For arbitrary $x_{1} \in K$ define the sequence $\left\{x_{n}\right\}$ iteratively by

$$
x_{n+1}:=x_{n}-\alpha_{n}(I-T) x_{n}, n \geq 1,
$$

where $\left\{\alpha_{n}\right\} \subseteq(0,1), \lim \alpha_{n}=0$ and $\sum \alpha_{n}=\infty$. Then, there exists a constant $d_{0}>0$ such that if $0<\alpha_{n} \leq d_{0},\left\{x_{n}\right\}$ converges strongly to the unique fixed point of $T$.

Proof. The proof is similar to the proof of Corollary 3.3 with the use of Theorem 3.6 instead of Theorem 3.2. 
Theorem 3.8. Let $E$ be a real uniformly smooth Banach space. Suppose $K$ is a closed convex subset of $E$ which is a nonexpansive retract of $E$ with $P$ as the nonexpansive retraction. Suppose $A: K \rightarrow E$ is a bounded uniformly accretive map with strictly increasing function $\psi:[0, \infty) \rightarrow[0, \infty)$ such that $\psi(0)=0$ and the solution $x^{*}$ of the equation $A x=0$ exists. For arbitrary $x_{1} \in K$, define the sequence $\left\{x_{n}\right\}$ iteratively by

$$
x_{n+1}:=P\left(x_{n}-\alpha_{n} A x_{n}\right), n \geq 1,
$$

where $\lim \alpha_{n}=0$ and $\sum \alpha_{n}=\infty$. Then, there exists a constant $d_{0}>0$ such that if $0<\alpha_{n} \leq d_{0},\left\{x_{n}\right\}$ converges strongly to the unique solution of $A x=0$.

Proof. As in the proof of Theorem 3.6 let $r$ be sufficiently large such that $x_{1} \in$ $B_{r}\left(x^{*}\right)$. Define $G:=\overline{B_{r}\left(x^{*}\right)} \cap K$. Then, since $A$ is bounded, we have that $A(G)$ is bounded. Let $M=\sup \{\|A x\|: x \in G\}$. As $j$ is uniformly continuous on bounded subsets of $E$, for $\epsilon:=\frac{\psi\left(\frac{r}{2}\right)}{2 M}$ there exists a $\delta>0$ such that $x, y \in D(T),\|x-y\|<\delta$ implies $\|j(x)-j(y)\|<\epsilon$. Set $d_{0}=\min \left\{\frac{r}{2 M}, \frac{\delta}{2 M},\right\}$.

Claim: $\left\{x_{n}\right\}$ is bounded. It suffices to show that $x_{n}$ is in $G$ for all $n \geq 1$. The proof is by induction. By our assumption $x_{1} \in G$. Suppose $x_{n} \in G$. We prove that $x_{n+1} \in G$. Assume for contradiction that $x_{n+1} \notin G$. Then, since $x_{n+1} \in K \forall n \geq 1$, we have that $\left\|x_{n+1}-x^{*}\right\|>r$. Thus we have the following estimates:

$$
\left\|x_{n+1}-x^{*}\right\|=\left\|P\left(x_{n}-\alpha_{n} A x_{n}\right)-P x^{*}\right\| \leq\left\|x_{n}-x^{*}-\alpha_{n} A x_{n}\right\|
$$

and hence

$$
\left\|x_{n}-x^{*}\right\| \geq\left\|x_{n+1}-x^{*}\right\|-\alpha_{n}\left\|A x_{n}\right\|>r-\alpha_{n} M \geq r-\frac{r}{2}=\frac{r}{2} .
$$

Set $r_{n}:=x_{n}-\alpha_{n} A x_{n}$. Then from (3.9), Lemma 2.1 and the above estimates we have that

$$
\begin{aligned}
& \left\|x_{n+1}-x^{*}\right\|^{2} \\
& \quad=\left\|P\left(x_{n}-\alpha_{n} A x_{n}\right)-P x^{*}\right\|^{2} \leq\left\|x_{n}-x^{*}-\alpha_{n} A x_{n}\right\|^{2} \\
& \quad \leq\left\|x_{n}-x^{*}\right\|^{2}-2 \alpha_{n}\left\langle A x_{n}, j\left(r_{n}-x^{*}\right)\right\rangle \\
& \quad=\left\|x_{n}-x^{*}\right\|^{2}-2 \alpha_{n}\left\langle A x_{n}, j\left(x_{n}-x^{*}\right)\right\rangle-2 \alpha_{n}\left\langle A x_{n}, j\left(r_{n}-x^{*}\right)-j\left(x_{n}-x^{*}\right)\right\rangle \\
& \quad \leq\left\|x_{n}-x^{*}\right\|^{2}-2 \alpha_{n} \psi\left(\left\|x_{n}-x^{*}\right\|\right)+2 \alpha_{n}\left\|A x_{n}\right\|\left\|j\left(r_{n}-x^{*}\right)-j\left(x_{n}-x^{*}\right)\right\| .
\end{aligned}
$$

Since $\left\|r_{n}-x_{n}\right\| \leq \alpha_{n}\left\|A x_{n}\right\| \leq \alpha_{n} M<\delta$ we have that $\left\|j\left(r_{n}-x^{*}\right)-j\left(x_{n}-x^{*}\right)\right\| \leq$ $\frac{\psi\left(\frac{r}{2}\right)}{2 M}$. Thus (3.10) gives that

$$
\left\|x_{n+1}-x^{*}\right\|^{2} \leq\left\|x_{n}-x^{*}\right\|^{2}-2 \alpha_{n} \psi\left(\frac{r}{2}\right)+\alpha_{n} \psi\left(\frac{r}{2}\right)<r^{2},
$$

i.e., $\left\|x_{n+1}-x^{*}\right\|<r$, a contradiction. Therefore $x_{n+1} \in G$. Thus by induction $\left\{x_{n}\right\}$ is bounded. Now we show that $x_{n} \rightarrow x^{*}$. Note that $r_{n}-x_{n} \rightarrow 0$ as $n \rightarrow \infty$ and hence by the uniform continuity of $j$ on bounded subsets of $E$ we have that

$$
\bar{\gamma}_{n}:=\left\|j\left(r_{n}-x^{*}\right)-j\left(x_{n}-x^{*}\right)\right\| \rightarrow 0 \text { as } n \rightarrow \infty .
$$

Let $\lambda_{n}:=\left\|x_{n}-x^{*}\right\|^{2}$ and $\gamma_{n}:=2 \alpha_{n} M \bar{\gamma}_{n}$. Then from inequality (3.10) we obtain that

$$
\lambda_{n+1} \leq \lambda_{n}-2 \alpha_{n} \bar{\psi}\left(\lambda_{n}\right)+\gamma_{n}, \text { where } \bar{\psi}(t):=\psi(\sqrt{t}) \text { and } \frac{\gamma_{n}}{\alpha_{n}} \rightarrow 0 \text { as } n \rightarrow \infty .
$$

Therefore, the conclusion of the theorem follows from Lemma 2.2. 
Corollary 3.9. Let $E$ be a real uniformly smooth Banach space. Suppose $K$ is a closed convex subset of $E$ which is a nonexpansive retract of $E$ with $P$ as the nonexpansive retraction. Suppose $T: K \rightarrow E$ is a bounded uniformly hemi-contractive map with strictly increasing function $\psi:[0, \infty) \rightarrow[0, \infty)$ such that $\psi(0)=0$. For arbitrary $x_{1} \in K$, define the sequence $\left\{x_{n}\right\}$ iteratively by

$$
x_{n+1}:=P\left(x_{n}-\alpha_{n}(I-T) x_{n}\right), n \geq 1,
$$

where $\lim \alpha_{n}=0$ and $\sum \alpha_{n}=\infty$. Then, there exists a constant $d_{0}>0$ such that if $0<\alpha_{n} \leq d_{0},\left\{x_{n}\right\}$ converges strongly to the unique fixed point of $T$.

Proof. The proof follows from Theorem 3.8.

If in Theorem 3.8 and Corollary 3.9, $0 \leq \alpha_{n}<1$ and the operator is self-map, the use of the projection operator $P$ will not be necessary. In fact, the following corollaries follow trivially.

Corollary 3.10. Suppose $E$ is a real uniformly smooth Banach space and $A$ : $E \rightarrow E$ is a bounded uniformly quasi-accretive map with strictly increasing function $\psi:[0, \infty) \rightarrow[0, \infty)$ such that $\psi(0)=0$. For arbitrary $x_{1} \in E$, define the sequence $\left\{x_{n}\right\}$ iteratively by

$$
x_{n+1}:=x_{n}-\alpha_{n} A x_{n}, n \geq 1,
$$

where $\left\{\alpha_{n}\right\}$ is a real positive sequence such that $\lim \alpha_{n}=0$ and $\sum \alpha_{n}=\infty$. Then, there exists a constant $d_{0}>0$ such that if $0<\alpha_{n} \leq d_{0},\left\{x_{n}\right\}$ converges strongly to the unique solution of the equation $A x=0$.

Corollary 3.11. Suppose $K$ is a closed convex subset of a real uniformly smooth Banach space E. Suppose $T: K \rightarrow K$ is a bounded uniformly hemi-contractive map with strictly increasing function $\psi:[0, \infty) \rightarrow[0, \infty)$ such that $\psi(0)=0$. For arbitrary $x_{1} \in K$, define the sequence $\left\{x_{n}\right\}$ iteratively by

$$
x_{n+1}:=x_{n}-\alpha_{n}(I-T) x_{n}, n \geq 1,
$$

where $\alpha_{n} \subseteq(0,1), \lim \alpha_{n}=0$ and $\sum \alpha_{n}=\infty$. Then, there exists a constant $d_{0}>0$ such that if $0<\alpha_{n} \leq d_{0},\left\{x_{n}\right\}$ converges strongly to the unique fixed point of $T$.

Remark 3.12. Recently, one of the authors (CEC) and Mutangadura [13] constructed an example of a Lipschitz pseudocontractive map defined on a compact convex subset of a Hilbert space into itself with a unique fixed point for which no Mann sequence converges. This example shows that for the iterative approximation of fixed points of Lipschitz pseudocontractions, the Mann iteration method does not always converge and so the Ishikawa iteration process (see, e.g., [19]) is certainly desirable. However, it is clear that whenever a Mann sequence converges, it is preferred to the Ishikawa process because of its simpler form. But, because the Ishikawa method was successfully applied to approximate fixed points of Lipschitz pseudocontractive maps, the following modification of it (which we shall refer to as Ishikawa-type) was introduced: If $K$ is a nonempty convex subset of a real normed linear space and $T: K \rightarrow K$ is a map, then for arbitrary $x_{0} \in K$, the sequence $\left\{x_{n}\right\}$ in $K$ is defined by

$$
x_{n+1}=\left(1-\alpha_{n}\right) x_{n}+\alpha_{n} T y_{n} ; y_{n}=\left(1-\beta_{n}\right) x_{n}+\beta_{n} T x_{n}, n \geq 0,
$$

where, (i) $0 \leq \alpha_{n}, \beta_{n}<1$ for all integers $n \geq 0$; (ii) $\lim \alpha_{n}=\lim \beta_{n}=0$; (iii) $\sum \alpha_{n}=\infty$. 
We emphasize the following observations:

(1) The condition $0 \leq \alpha_{n} \leq \beta_{n}<1$ in the Ishikawa scheme is replaced with (i) where $\alpha_{n}$ and $\beta_{n}$ are independent.

(2) The condition $\sum \alpha_{n} \beta_{n}=\infty$ is replaced with condition (iii).

It turns out, however, that if the Ishikawa-type sequence defined by (3.14) with conditions (i)-(iii) converges, then the Mann sequence $\left\{x_{n}\right\}$ defined by $x_{0} \in K$,

$$
x_{n+1}=\left(1-\alpha_{n}\right) x_{n}+\alpha_{n} T x_{n}, n \geq 0,
$$

where (a) $0 \leq \alpha_{n}<1$ for all integers $n \geq 0$; (b) $\lim \alpha_{n}=0$, (iii) $\sum \alpha_{n}=\infty$, converges. On the other hand, if the Mann sequence converges, by setting $\beta_{n} \equiv 0$ for all integers $n \geq 0$ in the recursion formulas (3.14), the Ishikawa-type sequence reduces to the simpler Mann sequence.

Thus, in this case, the Ishikawa-type sequence $\left\{x_{n}\right\}$ defined by equations (3.14) with conditions (i)-(iii), which has been studied extensively by various authors, is not needed. The simpler Mann-type sequence always converges in this case and is preferred.

Remark 3.13. It is known that if a theorem is proved using a Mann or Ishikawa sequence (without the so-called error terms (see, e.g., 21]) in the recursion formula), then the introduction of bounded error terms in the recursion formula does not lead to further generalizations. The computations are generally mere repetitions of those without error terms.

Remark 3.14. All our theorems and corollaries in this paper hold when the maps are set-valued, if such maps admit single-valued selections. In such cases each operator in our recursion formula is replaced with its single-valued selection. We omit the details. We remark in passing that a uniformly continuous pseudocontractive map cannot be set-valued (see, e.g., [22]).

Remark 3.15. As has been noted in the Introduction, our theorems and corollaries extend, unify, and generalize several important known results. In particular, Corollary 3.3 extends Theorem 2.1 of $[29$ from real smooth Banach spaces to real normed linear spaces and without the requirement that $T(D)$ be bounded. Theorem 3.6 extends Theorem 2.2 of 24 by dispensing with the boundedness condition imposed on the range of operator $A$. Corollary 3.7 extends Theorem 3.2 of [11] to the more general class of uniformly hemi-contractive maps. Theorem 3.8 and Corollary 3.9 extend Theorems 2 and 3 of [14], respectively, to a more general class of maps considered here.

\section{ACKNOWLEDGMENT}

The authors thank the referee for useful comments.

\section{REFERENCES}

[1] Alber, Ya., Reich, S.: An iterative method for solving a class of nonlinear operator equations in Banach spaces, Panamerican Math. J., Vol. 4, No. 2 (1994), 39-54. MR 95f:47089

[2] F. E. Browder, Nonlinear mappings of nonexpansive and accetive type in Banach spaces, Bull. Amer. Math. Soc. 73 (1967), 875-882. MR 38:581 
[3] S.-S. Chang, On Chidume's open questions and approximation solutions of multi-valued strongly accretive mapping equations in Banach spaces, J. Math. Anal. Appl. 216 (1997), 94-111.

[4] S.-S. Chang and K. K. Tan, Iteration process of fixed point for operators of monotone type in Banach spaces, Bull. Austral. Math. Soc. 57 (1998), 433-445. MR 99e:47083

[5] C.E. Chidume, Iterative construction of fixed points for multi-valued operators of the monotone type, Applicable Analysis 23 (1986), 209-218. MR 88e:47111

[6] C.E. Chidume, Iterative approximation of fixed points of Lipschitzian strictly pseudocontractive mappings, Proc. Amer. Math. Soc. 99 (1987), 283-288. MR 87m:47122

[7] C.E. Chidume, An iterative process for nonlinear Lipschitzian strongly accretive mapping in $L^{p}$ spaces, J. Math. Anal. Appl. 151 (1990), 453-461. MR 91k:47080

[8] C.E. Chidume, Approximation of fixed points of strongly pseudo-contractive mappings, Proc. Amer. Math. Soc. 120 (1994), 545-551. MR 94d:47056

[9] C.E. Chidume, Iterative solution of nonlinear equations of strongly accretive type, Math. Nachr. 189 (1998), 49-60. MR 99g:47141

[10] C.E. Chidume, Convergence theorems for strongly pseudocontractive and strongly accretive nonlinear maps, J. Math. Anal. Appl. 228 (1998), 254-264. MR 99h:47065

[11] C.E. Chidume and C. Moore, Steepest descent method for equilibrium points of nonlinear systems with accretive operators, J. Math. Anal. Appl. 245 (2000), 142-160. MR 2001a:47075

[12] C.E. Chidume and C. Moore. The solution by iteration of nonlinear equations in uniformly smooth Banach spaces, J. Math. Anal. Appl. 215 (1997), 132-146. MR 98m:47107

[13] C. E. Chidume and S. Mutangadura, An example on the Mann iteration methods for Lipschitzian pseudocontractions, Proc. Amer. Math. Soc. 129 (2001), 2359-2363. MR 2002f:47104

[14] C. E. Chidume, H. Zegeye and B. Ntatin, A generalized steepest descent approximation for the zeros of $m$-accretive operators, J. Math. Anal. Appl. 261 (1999), 48-73. MR 2000e:47080

[15] L. Deng, On Chidume's open questions, J. Math. Anal. Appl. 174 (1993), 441-449. MR 94b:47073

[16] L. Deng, Iteration processes for nonlinear Lipschitzian strongly accretive mappings in $L_{p}$ spaces, J. Math. Anal. Appl. 188 (1994), 128-140. MR 96f:47124

[17] L. Deng and X. P. Ding, Iterative approximation of Lipschitz strictly pseudocontractive mappings in uniformly smooth Banach spaces, Nonlinear Analysis 24 (1995), 981-987. MR 96a:47096

[18] J. C. Dunn, Iterative construction of fixed points for multivalued operators of the monotone type, J. Funct. Ann. 27 (1978), 38-50. MR 81f:47056

[19] S. Ishikawa, Fixed points by a new iteration Method, Proc. Amer. Math. Soc. 44 (1974), 147-150. MR 49:1243

[20] T. Kato, Nonlinear semi-groups and evolution equations, J. Math. Soc. Japan 19 (1967), 508-520. MR 30:1820

[21] L. Liu, Ishikawa and Mann iterative process with errors for nonlinear strongly accretive mappings in Banach spaces, J. Math. Anal. Appl. 194 (1995), 114-125. MR 97g:47069

[22] L. W. Liu and Y. Q. Li, On generalized set-valued variational inclusions, J. Math. Anal. Appl. 261 (2001), 231-240. MR 2002g:47142

[23] W. R. Mann, Mean value methods in iteration, Proc. Amer. Math. Soc. 4 (1953), 506-510. MR 14:998f

[24] C. Moore and B.V.C. Nnoli, Iterative solution of nonlinear equations involving set-valued uniformly accretive operators, Computers Math. Applic. 42 (2001), 131-140. MR 2002f:47145

[25] M.O. Osilike, Iterative solution of nonlinear equations of the $\psi$-strongly accretive type, $J$. Math. Anal. Appl. 200 (1996), 259-271. MR 97d:65032

[26] M.O. Osilike, Iterative construction of fixed points of multi-valued operators of the accretive type, Soochow J. Math. 22 (1996), 485-494. MR 97i:47122

[27] M.O. Osilike, Iterative construction of fixed points of multi-valued operators of the accretive type II, Soochow J. Math. 24 (1998), 141-146. MR 99e:47079

[28] B.E. Rhoades and L. Saliga, Some fixed point iteration procedures II, Nonlinear Analysis forum 6 (2001), 193-217. MR 2002f:47115

[29] S. Zhang, On the convergence problems of Ishikawa and Mann iteration process with errors for $\psi$-pseudo contractive type mappings. Appl. Math. Mechanics 21 (2000), 1-10. MR 2001c: 47080 
[30] X.L. Weng, Iterative construction of fixed points of a dissipative type operator, Tamkang J. Math. 23 (1992), 205-215. MR 94c:47077

[31] E. Zeidler, Nonlinear functional analysis and its applications, Part II: Monotone Operators, Springer-Verlag, 1985. MR 91b:47001; MR 91b:47002

The Abdus Salam International Centre for Theoretical Physics, Trieste, Italy

E-mail address: chidume@ictp.trieste.it

The Abdus Salam International Centre for Theoretical Physics, Trieste, Italy

E-mail address: habz@ictp.trieste.it 